artigO $] \begin{aligned} & \text { [RAFAELA NOROGRANDO ] } \\ & \begin{array}{l}\text { Doutora em Design e mestre em Antropologia Social e Cultural, trabalhou nove anos } \\ \text { como designer de calçados. Atualmente é docente de Design no ensino superior em }\end{array}\end{aligned}$ Portugal e divulga suas pesquisas no blog i-material, www.norogrando.wordpress.com. Professora na Universidade da Beira Interior e no Instituto Politécnico de Viseu. Pesquisadora associada ao ID+ Research Institute for Design, Media and Culture. E-mail: norogrando@gmail.com

\title{
Narrativas patrimoniais sobre moda: análise das temáticas expositivas e das escolhas museográficas
}

Patrimonial narratives: analysis of the thematic and

[resumo] Este artigo tem como objetivo a reflexão da construção histórica cultural que é feita sobre a moda e o design e apresenta uma planilha que atua como ferramenta de verificação das escolhas adotadas em exposições de moda. Utilizou-se de duas abordagens metodológicas: (1) uma foi quantitativa, com base na sinopse de exposições de moda realizadas ao redor do mundo. Esse procedimento resultou em um levantamento de dados de 459 exposições analisadas por suas escolhas temáticas. (2) Dessas exposições foram destacadas 29 para estudos de caso em uma abordagem qualitativa do contexto expositivo e escolhas conceituais. Teve-se o cuidado de abranger um número representativo de instituições (total de 11 museus) por caracteristicas distintas e abrangência geopolitica (seis paises diferentes). Como resultado, são apresentadas as temáticas das narrativas que vêm sendo feitas sobre a moda nos últimos anos (2008-2015), outras propostas que cabem ao universo da moda e do design e a confrontação das temáticas com as ferramentas de comunicação do espaço expositivo. Como conclusão, é verificada a predileção por algumas abordagens e defende-se a revisão desses padrões para uma expansão da construção patrimonial do universo da moda enquanto cultura material e imaterial.

\section{[palavras-chave}

exposições de moda; construção cultural; memória; museografia.

[abstract] This paper aims to reflect the cultural historical construction that is made about fashion and design. It presents a spread sheet that acts as tool of verification of the choices had been made in fashion exhibitions. It was used two methodological approaches: (1) quantitative: based on the synopsis of fashion exhibitions around of the world. This procedure resulted in a data collecting of 459 exhibitions analysed for its thematic choices. (2) qualitative: of these exhibitions were highlighted 29 for case studies to verify the context and conceptual choices. Careful to include a representative number of institutions have had it (total of 11 museums) by distinct features and geopolitical range (six different countries). As a result, this paper presented the themes have been made about fashion on exhibitions, focused in recent years (2008-2015), and other proposals regarding the world of fashion and design. Moreover, is conducted a confrontation with the thematic and the communication tools of the exhibition space. As conclusion the predilection for some approaches are verified and is defended it walk through of these standards for an expansion of the patrimonial construction of the universe of the fashion, as material and intangible culture. 


\section{Introdução}

A moda pode ser estudada como objeto, imagem, texto, prática, teoria ou conceito, por suas características abstratas ou materiais, especificas ou interdisciplinares. A moda pode ser vista por seus significados afetivos e pessoais, usos e hábitos, diferenças de gêneros e faixas de idades, e, assim, mais amplamente por uma visão de "cultura material de moda" (RIELLO, 2011). Nesse sentido, as possibilidades de narrativas expositivas são infinitas, e é importante que assim sejam para que a percepção desse universo venha a ser cada vez mais ampliada. No entanto, em investigação às abordagens museológicas sobre a exposição de traje/moda, percebe-se que ainda lentamente são trazidas ao público outras construções narrativas e históricas sobre a moda, ou mesmo sobre a roupa. Além disso, o conceito de vestir como cultura ainda está atrelado a padrões referencias e vinculado a uma tradição narrativa, por uma visão eurocentrista, autoral-capitalista, ou mesmo por sua superfície estética. Assim, a história da roupa acaba por ser eclipsada. Portanto, é pertinente defender projetos de curadorias que expandam a percepção do visitante para outras esferas da cultura material de moda.

0 processo expositivo de trajes em museus (e mesmo a história da moda) segue por uma tradição ligada à história da arte - conforme verificado nos espaços expositivos investigados - e já pontuado por outros autores. Isso quer dizer que é dada atenção a alguns atributos e não a outros, que estética tem mais relevância que processo, que há uma distância entre a já instituída dicotomia entre arte e ciência, e que as exposições de moda, em sua maioria, são apresentações de obras de arte.

Como defende Fallan (2010), se considerada somente a herança de um estudo e narrativa pela história da arte, a história do design é amputada, pois, segundo o autor, ela exige outras análises, outra forma de ser pesquisada e narrada. Ao encontro disso, Riello (2011) pontua três maneiras de estudar a história de moda, já que esta inclui objetos materiais e conceitos abstratos. Assim, expõe (1) a importância dos objetos em si, mas também (2) os conceitos a eles relacionados e, principalmente, (3) os significados a eles atribuídos na sua relação com a vida humana. Dessa maneira, por uma visão mais próxima à posição de designer do que de historiador, e concordando com a visão de Eilean Hooper-Greenhill, entende-se que as pessoas percebem melhor uma história quando esta é trazida mais próxima de suas "estratégias interpretativas e repertórios" (HOOPER-GREENHILL, 2000, p. 3).

\section{Exposições de moda: duas abordagens de pesquisa ${ }^{1}$}

Como base de estudo para a reflexão sobre a construção cultural que é feita sobre a história da moda foram realizadas duas abordagens metodológicas às ações expositivas do patrimônio de moda. A primeira, com caráter quantitativo, centrou-se na revisão e na classificação do maior número possivel de exposições quanto às suas temáticas. A segunda, qualitativa, foi realizada por pesquisa etnográfica nos locais e assumiu diversos critérios para a verificação do espaço narrativo.

Apesar do crescente surgimento de novos trabalhos relacionados com moda e museu, ainda resta muito a ser pesquisado. Nesse sentido, a busca por instituições que não fossem somente internacionalmente renomadas, mas também representativas por outros critérios, auxiliou na expansão da visão do cenário para um contexto global, e não apenas anglo-saxônico. Dessa maneira, os resultados das pesquisas realizadas contaram com uma amostra diversificada e representativa, e isso contribui para uma percepção mais realista do contexto globalizado.

Sobre a abordagem temática, foi considerado o trabalho de Horsley (2014) com uma pesquisa sobre exposições de moda de 1971 a 2013. Entre os anos de 2008 e 2014, foi feita uma revisão dessa listagem e foram incluídas outras exposições e instituições não contempladas por Horsley por questões de atualização e expansão geográfica (político-cultural). Esse trabalho resultou em uma análise global de 459 exposições realizadas ao redor do mundo - inventário publicado em Norogrando (2014). Estas foram classificadas por suas temáticas conforme foi possível verificar em diferentes documentos que as apresentavam - principalmente websites institucionais, artigos da revista Fashion Theory e catálogos de exposições. 
Para a segunda abordagem, quanto à construção cultural sobre a moda em contexto patrimonial e museológico, foi feita uma análise comparativa a estudos de casos investigados in loco. Essa pesquisa envolveu 11 instituições - nomeadamente: Museu Nacional do Traje, Museu do Design e da Moda, Museo del Traje, Victoria and Albert Museum, Museu Tèxtil i d'Indumentària, Museu do Traje de Viana do Castelo, Museo Nacional de la Historia del Traje, Fashion Museum, Fashion and Textile Museum, Museo de la Moda, Mode Museum - em seis países (Portugal, Espanha, Inglaterra, Argentina, Chile e Bélgica), somando 11 exposições permanentes e 18 exposições temporárias que decorreram entre 2011 e $2015^{2}$. Nesse processo de investigação sobre 0 design de exposição foram utilizadas as seguintes categorias de análise: (1) conceitos de comunidade; (2) conceitos de espaço e discurso; (3) narrativas; (4) ferramentas de comunicação; (5) a questão do corpo; e (6) outras atividades. Aqui não será explorado todo o resultado dessa abordagem, como já foi feito em outras publicações - de maneira geral em Norogrando e Mota (2014) e somente sobre a questão do corpo em Norogrando (2015) -, mas apresenta-se o avanço desse trabalho na concepção de uma ferramenta de análise e construção de conhecimento ou concepção expositiva sobre a história da moda.

\section{Breves considerações quanto à construção cultural sobre a moda}

A moda faz parte da cultura visual, do patrimônio da cultura material e também da cultural imaterial, assim, conjuga em si mais de uma apreciação, é constituída por seu aspecto visual, e compreendida por meio da visão, pela pele, pelo corpo em movimento e pelo envolvimento com um contexto. No entanto, existe uma predileção de temas aos quais a moda, por meio de seus objetos de vestuário, é exposta por museus ou outras instituições, conforme foi detalhado em Norogrando e Mota (2014).

De acordo com estudos anteriores quanto ao processo de inventário dos museus (NOROGRANDO, 2011), pode-se verificar que muito do que é considerado em termos de valor no sistema de mercado acaba por permanecer com esse atributo quando é averiguada a sua integração a uma coleção patrimonial. No desenvolvimento de uma exposição muito do que será comunicado também, inevitavelmente, passará por esse filtro de valor.

Em uma entrevista de 2014, Valerie Steele, diretora e curadora chefe do M-FIT, reforçou que a missão no museu é de avançar com o conhecimento de moda para tentar que as pessoas "levem a sério a moda, reconhecendo que ela pode ser muito divertida, mas fundamentalmente que encarem a moda seriamente como uma forma cultural que é significativa" e também que "há um objetivo de desafiar a banalização de mitos sobre moda para tentar mostrar às pessoas que a moda é mais complicada do que elas pensam" (BLACK, 2014). Esse entendimento também é reforçado por Clive Dinot (2014) quando discute o conceito de museu de design e construção histórica sobre o design de forma a que esses contribuam para reflexões e inovações que atendam ao presente e ao futuro das ações humanas frente aos seus contextos e responsabilidades.

É com esse intuito que as análises realizadas corroboram para a obtenção de uma visão alargada sobre o que vem sendo construído como narrativas da história da moda. As ações museológicas vão solidificando os conceitos desse universo, e algumas narrativas são patrimonializadas, sendo abrangentes ou não. Muito do que foi feito nos processos de inventário não pode ser reestruturado, mas as histórias que são contadas por meio dos objetos patrimoniais e de ferramentas de comunicação são passiveis de constante atualização e contextualização. Por esse motivo, apresenta-se uma ferramenta de revisão dessa construção de conhecimento, a qual é composta por temas e estratégias de curadoria mais utilizadas na atualidade, outras duas perspectivas e ferramentas museográficas que incidem na expansão da comunicação de moda e de sua construção enquanto patrimônio cultural. 


\section{Exposições de traje/moda: práticas de comunicação sobre o tema}

No que diz respeito às exposições de traje/moda, houve mudanças significativas no conceito e na concepção das mesmas. Conforme Melchior (2014), as exposições de roupas podem ser percebidas por sequência de fases conceituais: inicialmente, centradas no objeto enquanto matéria têxtil e, atualmente, o mesmo objeto assume uma perspectiva mais contextual - uma alteração natural promovida por estudos sobre a moda como fenômeno social e por contextos gerais e específicos. Entretanto, conforme as análises globais e os estudos de casos, as ações que fogem a um modelo narrativo padrão ainda são pontuais, o que poderia ser ampliado de acordo com 0 próprio universo da moda.

Nesse sentido, este artigo apresenta uma planilha formulada para a revisão da construção cultural sobre a história da moda em exposições sobre a temática. Nela é possivel ponderar sobre as diversas abordagens conceituais e experienciais a respeito da moda. Para tanto, foram cruzadas as informações retiradas das pesquisas anteriormente mencionadas para a formulação de um conjunto de dados, nomeadamente: temas para exposições de moda e modelos de percepção para 0 visitante em espaço expositivo.

\section{Temáticas expositivas}

Por meio da avaliação de 459 exposições realizadas nos últimos sete anos e da análise desenvolvida por Horsley (2014a), foi feita uma listagem de temas utilizados em exposições sobre a moda (Tabela 1). Além disso, foram acrescidos alguns tópicos relevantes e intrinsecos ao universo da moda, tendo em conta uma análise reflexiva sobre este, conforme já defendia Steele (1997) quanto as atuações do M-FIT.

Tabela 1: Temáticas de exposições de traje/moda (realizadas e sugeridas).

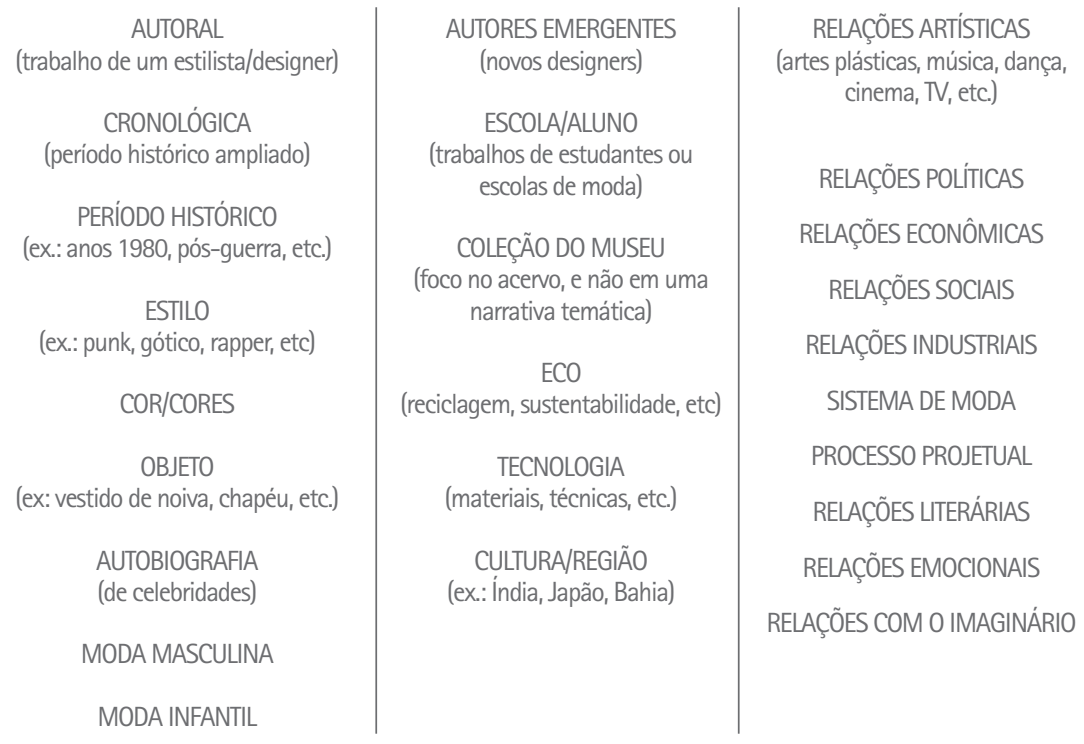

\section{Experiência expositiva e museografia}

Para os modelos de percepção para o visitante de uma exposição foram adotados os conceitos de Lord e Lord (2002) em relação com os de Dernie (2006) e Hughes (2010). No entanto, para essa proposta não foi considerado relevante manter o modelo de "descobrimento" indicado por Lord e Lord (2002), por entender que tal desdobramento tornaria a tabela muito detalhada e de abordagem pouco eficiente. Dessa maneira, foram utilizados somente os modelos de contemplação, compreensão e interação. É também pertinente ponderar que os modelos de "contemplação" e "compreensão" são divididos por uma linha tênue, pois, conforme verificado, a relação 
entre um e outro é por vezes estabelecida no decorrer do percurso das exposições de moda. Isso ocorre não por uma estruturação de diorama (da relação entre o objeto e 0 contexto), mas principalmente pela relação do objeto com um tema.

Além do cruzamento por modelo expositivo, foram delimitadas algumas atuações possiveis nos espaços museológicos por ferramentas de comunicação, como é possível verificar na Tabela 2.

\section{Tabela 2: Modelos de percepção para o visitante de exposição}

\begin{tabular}{|c|c|c|}
\hline \multirow[t]{3}{*}{ CONTEMPLAÇÃO } & OBJETO & Objeto como centro, foco em sua representatividadde plástica/estética. \\
\hline & CONJUNTO & Conjunto de objetos que formam uma instalação, conjunto temático \\
\hline & DOC & $\begin{array}{l}\text { Apresentação correlacionada de outros documentos referentes aos } \\
\text { objetos de vestuário, tais como: fotografias em revista de moda, } \\
\text { desfiles, propagandas, fotografias pessoais/privadas, músicas e filmes } \\
\text { relacionados ao tema da exposição ou produzidos para tal. }\end{array}$ \\
\hline \multirow{4}{*}{ COMPREENSSÃO } & D-PM & $\begin{array}{l}\text { Depoimentos de profissionais de museu relacionados com a } \\
\text { exposição ou com a pesquisa e a investigação dos objetos e contextos } \\
\text { apresentados. }\end{array}$ \\
\hline & D-P & $\begin{array}{l}\text { Depoimentos de profissionais relacionados com a concepção e a } \\
\text { produção de objetos em exposição. }\end{array}$ \\
\hline & $\mathrm{D}-\mathrm{U}$ & $\begin{array}{l}\text { Depoimentos de pessoas que consumiram, utilizaram ou tiveram alguma } \\
\text { relação com os objetos em suas fases pós-processo industrial. }\end{array}$ \\
\hline & O-VESTIR & Objetos para serem vestidos pelos visitantes da exposição. \\
\hline \multirow[t]{3}{*}{ INTERAÇÃO } & O-TOCAR & Objetos para serem tocados. \\
\hline & DIG & Informações ou interações por ferramentas digitais. \\
\hline & JOG & Jogos e outras interações lúdicas. \\
\hline
\end{tabular}

Para auxiliar a verificação de temáticas expositivas e a relação dessas com os modelos de percepção disponibilizados aos visitantes, foi concebida uma terceira tabela. Esta é apresentada na sequência deste artigo e culmina a proposta de revisão dos modelos expositivos e a consequente construção cultural e histórica sobre o patrimônio de moda.

\section{Patrimônio em construções narrativas: temáticas x experiências}

Existe uma preponderância na escolha de temas e fórmulas expositivas para a apresentação de objetos da cultura material de moda. Isso já foi questionado por alguns autores (RIELLO e MCNEIL, 2010) e confirmado pelas análises apresentadas em publicações anteriores e pela pesquisa realizada por Horsley (2014a).

Para evidenciar essas constatações sobre uma predileção de temáticas combinadas a elementos de comunicação no espaço museográfico, foram distribuidos na Tabela 3 os 29 estudos de caso pesquisados. É pertinente mencionar que nem sempre uma exposição é conduzida por uma única caracteristica e, por esse motivo, há uma repetição dos números das exposições por mais de um tópico, a fim de melhor traduzir nessa planilha a diversidade e complexidade trabalhada nos espaços investigados.

Essa visão de temáticas e modelos expositivos traz à reflexão as escolhas de curadoria, já que, por vezes, poderia ser questionado se elas não estão sendo induzidas por conceitos preestabelecidos ou por inventários que estiveram em detrimento a conceitos ou necessidades de um dado período - conceitos esses muitas vezes trabalhados pela própria moda. No entanto, mesmo que sejam abordadas narrativas sobre a moda e não sobre a indumentária por uma perspectiva mais ampliada do vestir, o sistema de moda não deveria ser questionado? Será que por vezes o ciclo de consagração não poderia ser usurpado? Esses questionamentos foram levantados anteriormente por outras abordagens e retornam aqui à reflexão devido à composição da tabela, na 
qual fica evidente a recorrência de alguns temas em detrimento de outros, além das abordagens preferenciais ao contexto museográfico.

Por meio da composição visual formada na Tabela 3 é possivel verificar uma maior incidência de um modelo de apresentação contemplativa e um pouco menos do modelo de percepção compreensiva. Já um modelo interativo ou temáticas mais reflexivas sobre o contexto da moda ou da indumentária são pouco explorados, conforme se percebe nos dados apresentados nessa investigação e por outros autores.

Tabela 3: Estrutura de comunicação de conhecimento de moda em exposições museológicas

\begin{tabular}{|c|c|c|c|c|c|c|c|c|c|c|}
\hline & & & & ERIEN & EXY & SITIV & & & & \\
\hline & CONTEM & AÇÃO & & IPREENS & & & INTER & Ç̧̃o & & \\
\hline & OBJETO & CONJUNTO & DOC & D-PM & D-P & D-U & 0-VESTIR & 0-TOCAR & DIG & JOG \\
\hline AUTORAL & & & & & & & & & & \\
\hline CRONOLÓGICA & & & L & & & & & & 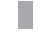 & \\
\hline PERIODO HISTÓRICO & & & & & & & & & & \\
\hline ESTILO & & & & & & & & & & \\
\hline COR & & & & & & & & & & \\
\hline OBJETO & & & $\square$ & & & & & & & \\
\hline AUTOBIOGRAFIA & & & & & & & & & & \\
\hline MASCUUINA & & & & & & & & & & \\
\hline INFANTIL & & & & & & & & & & \\
\hline AUTORES EMERGENTES & & & & & & & & & & \\
\hline ESCOLA/ALUNO & & & & & & & & & & \\
\hline COLEÇÃO DO MUSEU & & & & & & & & & & \\
\hline ECO & & & & & & & & & & \\
\hline TECNOLOGIA & & & & & & & & & & \\
\hline RELAÇÕES ARTISTICAS & & & & & & & & 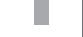 & - & \\
\hline CULTURA/REGIÃO & & & & & & 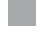 & & & L & \\
\hline RELAÇÕES POLITICAS & & & & & & & & & & \\
\hline RELAÇÕES ECONÔMICAS & & & & & & & & & & \\
\hline RELAÇÕES SOCIAIS & & & & & & & & & & \\
\hline RELAÇÕES INDUSTRIAIS & & & & & & & & & & \\
\hline SISTEMA DE MODA & & & & & & & & & & \\
\hline PROCESSO PROJETUAL & & & & & 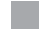 & & & & & \\
\hline RELAÇÕES LITERÁRIAS & & & & & & & & & & \\
\hline RELAÇÕES EMOCIONAIS & & & & & & 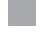 & 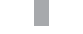 & & & \\
\hline RELAÇÕES COM O IMAGINÁRIO & & & & & & & & & & \\
\hline
\end{tabular}

$\mathrm{FM}^{*} \mathrm{~A}$ seção com objetos para prova está à parte das narrativas, não pertence às exposições investigadas no Fashion Museum.

A abordagem expositiva por narrativa cronológica é representativa nessa amostragem por apresentar muitos estudos de exposições permanentes, que costumam ser mais didáticas em uma representação de períodos ou estilos sequenciais de uma linha histórica eurocentrista. Além disso, é possivel verificar uma concentração de exposições relacionadas a uma temática apoiada em período histórico, e que nos estudos de caso centraram-se na década de 1980.

Essa amostragem também refletiu a representatividade de décadas da temática expositora por autoria. Esse preterido tema, por meio da exibição de master pieces da carreira de um grande criador, é uma consequência natural de abordagem retrospectiva das passarelas de moda. Outra temática que se destaca nessa coleta é a relação cultural/regional, pois, além das exposições que tiveram essa abordagem como temática - "Boro" (15) e "Exotismo" (25) -, estão relacionadas as exposições permanentes de museus voltados para a sua cultura local, como é o caso do Museu do Traje de Viana do Castelo e o MNHT, de Buenos Aires. Já a relação com celebridades em uma narrativa autobiográfica, também sinalizada na pesquisa de Horsley (2014a, 2014b), aparece como fio condutor de duas narrativas e também como critério para a ação de referenciar documentos relacionados com objetos em outras exposições.

Como evidência de uma mudança na perspectiva das exposições, percebe-se a incidência de algumas por uma classificação mais complexa da narrativa, são abordagens mais reflexivas e relacionadas a outros contextos que fogem aos padrões já sinalizados. Ainda assim, por meio da apreciação dos dados, nota-se um grande vazio 
na Tabela 3 em relação a alguns temas. Isso não ocorre em virtude do tamanho da amostragem, pois o resultado é o mesmo se compararmos com as ações analisadas nas 459 exposições dos últimos sete anos. Por exemplo, as narrativas centradas na relação da moda com tecnologia, indústria ou sustentabilidade também não são habituais. Essa constatação também acaba por evidenciar a moda por seu atributo artístico, o que permite perceber melhor as construções expositivas ligadas ao modelo de contemplação (relacionado aos museus de arte) perante os modelos interativos utilizados principalmente por centros de ciência.

É importante ressaltar que aqui não se tem a pretensão de induzir essas tabelas como fontes primárias para a estruturação de comunicações sobre a moda. Pelo contrário, parte-se do princípio de que deve existir a liberdade de escolha dos curadores frente as diversas possibilidades narrativas e recursos que disponibilizam. Nesse sentido, a Tabela 3 foi concebida com o intuito de promover uma verificação das escolhas para que se possa transpor a fórmula comum de narrativas produzidas nos últimos 43 anos sobre a história da moda. A correlação das listagens que foram elaboradas (tabelas 1 e 2 ) atua como um revisor para novas abordagens com base nos objetos e universos temáticos da moda/traje, e até mesmo do design em geral. Da mesma maneira, com a identificação das ferramentas de comunicação do design de exposição e a correlação dessas informações - temas x percepção do visitante + ferramentas de comunicação -, evidenciam-se as diversas possibilidades de como contar histórias sobre a moda e o design.

\section{Narrativas sobre a moda: que sejam muitas, que venham outras!}

Uma exposição museológica é visitada por diferentes públicos, e uma diversificação na maneira de contar e apresentar uma história pode dar respostas às diferentes expectativas das pessoas, além de proporcionar outros olhares e experiências sobre o universo da moda. De acordo com a Tabela 1, existe uma diversidade de temáticas narrativas como também há uma série de estratégias museográficas (Tabela 2) que podem ser empregadas na concepção das exposições com o intuito de almpliar o entendimento sobre esse universo. Como exemplo, destaca-se a humanização da representação narrativa desses objetos com vídeos ou holografias, nos quais eles são apresentados em uso/movimento, ou ainda, a disponibilização de réplicas para serem vestidas e outros elementos que promovam a percepção tátil. Como estratégia museográfica também se pode apresentar a reprodução dos processos de desenvolvimento/produção/divulgação dos objetos ou incluir elementos adjacentes a esses (modelagens, croquis, publicidade); alterar o olhar sobre o objeto com outros ângulos, do avesso à ampliação de detalhes; ou, ainda, como exemplo das infinitas possibilidades que podem ser empregadas, o visitante pode ser conduzido a uma imersão em paisagens sonoras e narrativas pessoais que podem causar estranheza ou identificação, mas sempre uma experiência pontual de aprendizado e/ou entretenimento.

Nos países onde a cultura de design e design de moda já é intrínseca ao cotidiano em uma natural valorização de suas atuações, a escolha de uma narrativa ou outra pode ter pouco efeito a curto prazo. No entanto, principalmente em situações nas quais ainda existe a necessidade de consolidação de atividades ligadas ao design, a presença da instituição museológica como agente comunicativo do setor a um público ampliado é de grande valia. Dessa forma, somente a narrativa estética, em uma dinâmica de valorização patrimonial/comercial, deixa de apresentar todo um contexto de concepção, produção, uso e descarte que poderia contribuir para uma consciência mais ampla da cultura material na atualidade e da herança que isso representa, não somente como fruto da criatividade humana, mas também de suas intervenções nos meios social e físico.

Por fim, entende-se que as ações institucionais podem atuar em uma dinâmica diferente a cada ciclo de exposições, de forma a apresentar ao público diversas narrativas e novas conexões acerca do universo temático traje/moda. Dessa maneira, 
fica evidente a relevância das tabelas - principalmente da 3 - como ferramenta de reflexão e auxílio na verificação de propostas narrativas sobre um universo amplo e complexo, que vai além de autores e períodos históricos específicos. Além disso, também é possivel visualizar uma aproximação entre a história, a prática do design e as relações culturais e imateriais desses objetos materiais patrimonializados.

\section{Conclusão}

0 que se constrói sobre o que é moda, sobre o que é design?

Este artigo apresentou uma síntese dos padrões expositivos sobre a moda e a proposta de verificação da comunicação e interpretação de objetos da cultura material, consequentemente a relação destes com pessoas e contextos. Assim, foram expostos contributos diretos para atuação ou mesmo reflexão de ações expositivas sobre a moda e o design.

A ferramenta de análise apresentada (Tabela 3) é válida como mecanismo que permite explicitar as diferentes abordagens que podem ser feitas ao universo da moda, e como elemento auxiliar para a ponderação das escolhas de curadoria na perspectiva de construção de memória e existência.

As novidades e propostas que foram apresentadas em Norogrando (2015a), uma das quais foi destacada aqui, servem como guia para práticas efetivas, como objeto de reflexão, instigam outras abordagens de estudo por diferentes disciplinas e a reflexão em contexto prático a colocar em causa cada escolha institucional.

[Recebido em: 11/08/2015]

[Aprovado em: 14/09/2015]

\section{NOTAS}

[1] Esta publicação teve origem no trabalho realizado para a tese de doutorado da autora: Exposições museológicas. A Moda por narrativas, experiências e conexões, a qual foi defendida em março de 2015, na Universidade de Aveiro (Portugal), e teve a investigação financiada por: FCT - (Fundação para a Ciência e a Tecnologia), Fundo Social Europeu, POPH (Programa Operacional Potencial Humano) e ID+ (Instituto de Investigação em Design, Media e Cultura).

[2] Por uma questão de conceito e concepção, apresentam-se de maneira separada as exposições investigadas.

Exposições permanentes:

Museu Nacional do Traje: (1) 0 Traje em Portugal. Do século XVIII à Contemporaniedade

Museu do Design e da Moda: (2) Único e múltiplo. 2 Séculos de Design. (2011-2014), (3) a mesma exposição após renovação integral inaugurada em 3/4/2014 e (4) Ante-Estreia - Flashes da Coleção (2009-2011).

Museo del Traje-CIPE: (5) História cronológica e (6) Área pedagógica.

V\&A: (7) Fashion Galleries (desde 5/2012).

Museu Tèxtil i d'Indumentària: (8) El cuerpo vestido (2008-2012, renovação integral inaugurada em 12/2014).

Museu do Traje de Viana do Castelo: (9) exposição permanente.

Museo Nacional de la Historia del Traje: (10) La Moda en el Rio del Plata e (11) História cronológica.

Exposições temporárias:

Museu Nacional do Traje: (12) Uma coleção coMnexo: o papel do doador (5 a 12/2014).

MUDE: (13) Morte ao Design! Viva o Design! (10/2011 a 1/2012), (14) Com esta Voz me Visto (11/2012 a 04/2013), (15) Japão a Cru. BORO: o tecido da vida (10/2014 a 2/2015).

VEtA: (16) Yohji Yamamoto (3 a 7/2011), (17) Ballrowns: British Glamour since 1950 (5/2012 a 6/2013), (18) Hollywood Costume (10/2012 a 1/2013), (19) Wedding Dresses 1775- 2014 (5/2014 a 3/2015).

Fashion Museum: (20) What Will She Wear? (2/2011 a 1/2012), (21) Behind the Scenes at the FM. The Historic Collection (inaugurada em 1/2011), (22) Daywear - 20th Century (inaugurada em 1/2011), (23) Top Trends. Spring Summer 2011.

Fashion and Textile Museum: (24) Tommy Nutter, Rebel on the Row (5 a 10/2011).

Museo Nacional de la Historia del Traje: (25) Exotismo. La influencia de Oriente en Occidente en la Moda (12/2010 a 11/2011); (26) Los 80's. Extravagantes y Glamorosos (6/2011 a 2012).

Museu Tèxtil i d'Indumentària: (27) Qué me pongo? El guardarropa de Maria Brillas por Pedro Rodriguez (3 a 9/2011).

Museo de la Moda: (28) Volver a los 80 (parte II) (4/2011 a 12/2012).

MoMu: (29) Living Fashion: Women's Daily Wear 1750-1950. From the Jacoba de Jonge Collection (3 a 8/2012). 
Nota-se que algumas exposições, principalmente as do Fashion Museum, deveriam ser classificadas como exposiç̃es permanentes devido ao seu ampliado tempo de duração. Entretanto, tais exposições foram inicialmente divulgadas como temporárias e, dessa maneira, na época da investigação, foram tratadas como tal. Acredita-se que sua concepção partiu dessa efemeridade expositiva e que posteriormente a instituição tenha optado por mantê-las ativas para o público por um periodo maior do que o programado. Situação inversa ocorre com a denominada exposição permanente do Museu do Design e da Moda em Lisboa, pois em cinco anos já foi alterada três vezes. Nesse caso, também se optou por adotar a classificação feita pelo museu e entende-se que o curto periodo de duração das exposições seja em detrimento de uma estratégia institucional, o que não invalida o caráter atribuído nesta pesquisa.

\section{REFERÊNCIAS}

BLACK, Renata M. Reconstructing the Meaning of Fashion. HuffPost Impact. Disponivel em: <http:// www.huffingtonpost.com/renata-m-black/reconstructing-the-meanin_b_5030400.html>. Acesso em: 13 abr. 2014.

DERNIE, David. Exhibition Design. London: Laurence Publishing King, 2006.

DINOT, Clive. Is There an Ethical Role for the History of Design? Redeeming Through History the Possibility of a Human World. In: Proceedings of 9th International Committee [on] Design History and Design Studies - ICDHS 2014: tradition, transitions, trajectories: major or minor influences?. Congresso e livro de atas coordenado por Helena Barbosa e Anna Calvera. Aveiro: UA Editora, 2014.

FALLAN, Kjetil. Design History:Understanding Theory and Method. New York, London: Bloomsbury, 2010.

HOOPER-GREENHILL, Eilean. Museums and the Interpretation of Visual Culture, Museum Meanings. London: Routledge, 2000.

HORSLEY, Jeffrey. An Incomplete Inventory of Fashion Exhibition Since 1971. In : CLARK, Judith; LA HAYE, Amy de. (eds.). Exhibition Fashion: Before and After 1971, Londres, New Haven: Yale, 2014a. p. 169-245.

Autobiography as a Proposed Approach to a Fashion Exhibition. In: MELCHIOR, M. R.; SVENSSON, B. (eds.). Fashion and Museums: Theory and Pratice. London, New York: Bloomsbury Academic, 2014b. p. 180-196.

HUGHES, Philip. Exhibition Design. London: Laurence King Publishing, 2010.

LORD, Barry; LORD, Gail Dexter. The Manual of Museum Exhibitions. Walnut Creek: Altamira Press, 2002

MELCHIOR, Marie Riegels. Introduction: Understanding Fashion and Dress Museology. In: MELCHIOR, M. R.; SVENSSON, B. (eds.). Fashion and Museums:Theory and Pratice. London, New York: Bloomsbury Academic, 2014. p. 1-18.

NOROGRANDO, Rafaela. Como é formado o patrimônio cultural. Estudo museológico em Portugal na temática Traje/Moda. Dissertação (Mestrado em Antropologia Social e Cultural), Departamento de Ciências da Vida, Universidade de Coimbra, Coimbra, Portugal, 2011.

NOROGRANDO, Rafaela. Fashion Exhibition/ Exposições de Moda. In: i-material, wordpress, 2014. Disponivel em: <https://norogrando.wordpress.com/2014/06/12/fashion-exhibition- exposicoes-demoda/>. Acesso em:10 ago. 2015.

NOROGRANDO, Rafaela; MOTA, João A. Narratives of Fashion:What Becomes Heritage? What Turns Into History? [Narrativas da moda: 0 que fica de patrimônio? 0 que vira história?] CIMODE Book of Proceedings, Milão, ISBN 978886493 0275, 2014. Disponivel em: <http://www.eko.polimi.it/public/files/ cimode2014/CIMODE\%202014_PROCEEDINGS.pdfs.

NOROGRANDO, Rafaela. Narrativas, experiências e conexões. In: Processos de Musealização: um seminário de investigação internacional. Atas do seminário, editado por Alice Semedo, Sandra Senra e Teresa Azevedo, 2015, p. 518-539. Disponivel em: <http://ler.letras.up.pt/uploads/ficheiros/13459.pdf>. <http://ler.letras.up.pt/uploads/ficheiros/13514.pdfs. Acesso em: 10 ago. 2015.

RIELLO, Giorgio; MCNEIL, Peter. (eds.). The Fashion History Reader: Global Perspectives. Oxon, New York: Routledge, 2010.

RIELLO, Giorgio. The Object of Fashion: Methodological Approaches to the History of Fashion. Aesthetics \& Culture, v. 3, 2011. Disponivel em: <http://www.aestheticsandculture.net/index.php/jac/article/ view/8865>. Acesso em: 15 abr. 2014

STEELE, Valerie. Exhibition Review: Two by Two. The Metropolitan Museum of Art. Fashion Theory. The Journal of Dress, Body \& Culture, v. 1, n. 1, 1997, p. 105-110. 